\title{
Fabrication of Highly Rough Ag Nanobud Substrates and Surface-Enhanced Raman Scattering of $\lambda$-DNA Molecules
}

\author{
Chuyun Deng, Wanyun Ma, and Jia-Lin Sun \\ State Key Lab of Low-Dimensional Quantum Physics, Department of Physics, Tsinghua University, Beijing 100084, China \\ Correspondence should be addressed to Jia-Lin Sun, jlsun@tsinghua.edu.cn
}

Received 18 May 2012; Accepted 6 August 2012

Academic Editor: Steve F. A. Acquah

Copyright () 2012 Chuyun Deng et al. This is an open access article distributed under the Creative Commons Attribution License, which permits unrestricted use, distribution, and reproduction in any medium, provided the original work is properly cited.

Raman scattering signals can be enhanced by several orders of magnitude on surface-enhanced Raman scattering (SERS) substrates made from noble metal nanostructures. Some SERS substrates are even able to detect single-molecule Raman signals. A novel silver nanobud (AgNB) substrate with superior SERS activity was fabricated with a solid-state ionics method. The AgNB substrate was formed by tightly collocated unidirectional $100 \mathrm{~nm}$ size silver buds, presenting a highly rough surface topography. Distinct SERS signals of single $\lambda$-DNA molecules in water were detected on AgNB substrates. AgNB substrates were compared with disordered silver nanowire $(\mathrm{AgNW})$ substrates manufactured by the same method through the SERS detection of $\lambda$-DNA solutions. This original AgNB substrate provides a reliable approach towards trace analysis of biomacromolecules and promotes the utilization of the SERS technique in biomedical research.

\section{Introduction}

Raman scattering is a characterization technique that provides fingerprint recognition by molecular vibrational and rotational energy levels indicated by the Raman peaks. Raman spectroscopy is especially suitable for biomedical studies because of its advantages in nondestructive detection, rich configuration information, easy sample preparation, and freedom from interference by water. Many applications of Raman spectroscopy in DNA, RNA, and protein research have been reported [1-5]. In recent decades, noble metal nanostructures have been discovered to have good Raman scattering enhancement ability. According to electromagnetic theories, external light can polarize free electrons on noble metal nanostructured surfaces and thereby cause vibration of the entire free surface electrons. When the frequency of external light matches that of the surface electron vibration, local charge distribution will be affected and the local field will be strongly enhanced due to surface plasmon resonance (SPR) phenomenon. For molecules adsorbed on such noble metal nanostructures, their Raman signals will be markedly intensified by SPR effects [6]. It was reported that silver nanostructure surfaces exhibited surface-enhanced Raman spectra of adsorbed molecules by several orders of magnitude and, in some cases, singlemolecule Raman signals could be detected. Hence, surfaceenhanced Raman scattering (SERS) substrates based on silver nanostructures have been widely employed in chemical and biomedical detection [7-14]. A large amount of outstanding metal nanostructures have been fabricated by our solidstate ionics method [15-17]. Among them a highly rough unidirectional silver nanobud (AgNB) structure has excellent SERS activity. In this work, the SERS activity of AgNBs is established by detecting the Raman signal of $\lambda$-DNA on $\mathrm{AgNB}$ substrate at the single-molecule level.

\section{Materials and Methods}

2.1. Fabrication of $A g N B$ Structures. Our solid-state ionics method is based on the ion-conducting ability of superionic conductor thin films that are comparable to molten salts or electrolyte solutions. The superionic conductor thin film was designed as the medium to transport metal ions between metal electrodes and generate a directional ionic current. Metal atoms at the anode were ionized by the external direct current (DC) electric field and transported to the cathode through the superionic conductor thin film to grow into various nanostructured materials [15-17]. 
The preparation process for highly rough silver nanostructures is shown in Figure 1. Thin silver films of $1 \mu \mathrm{m}$ thickness were deposited onto two sides of a clean quartz substrate to serve as the electrodes. The interelectrode distance was $8 \mathrm{~cm}$. A piece of $\mathrm{RbAg}_{4} \mathrm{I}_{5}$ superionic conductor thin film, about $400 \mathrm{~nm}$ thick with a $0.12 \Omega^{-1} \mathrm{~cm}^{-1}$ ionic conductivity at room temperature, was deposited onto the whole surface of the quartz substrate as the ion-conducting medium [17]. All deposition processes were conducted at room temperature and $10^{-4} \mathrm{~Pa}$ vacuum. The external DC electric field with a constant electric current was provided by a SourceMeter (Keithley 2400, USA). Silver atoms at the anode were ionized and transported to the cathode to grow into AgNB structures through the $\mathrm{RbAg}_{4} \mathrm{I}_{5}$ thin film, while electrons were transported to the cathode through external conducting wires. After 3 days, silver nanostructures of square centimeter size were obtained in the cathode area. Such silver nanostructures were composed of tightly collocated $100 \mathrm{~nm}$ sized units as revealed by scanning electron microscopy (SEM) (Figure 2(a)). The $100 \mathrm{~nm}$ sized units were arranged like nanoscale buds growing in the same direction, leading to a highly rough surface topography, and these silver nanostructures were termed silver nanobuds (AgNBs).

The external constant electric current or, rather, the ionic current density in the $\mathrm{RbAg}_{4} \mathrm{I}_{5}$ thin film is the crucial parameter in the fabrication of silver nanostructures. The growth of silver nanostructures is maintained by continuous and stable ionic current as a result of the external constant electric field. Different nanostructures will be obtained as the intensity of external electric current changes. The external electric current was $12 \mu \mathrm{A}$ in the growth of AgNBs with unidirectional and dense configuration (Figure 2(a)). However, when the external electric current was decreased to $3 \mu \mathrm{A}$, disordered and loose silver nanowires (AgNWs) were obtained (Figure 2(b)). The AgNBs were made of silver according to energy dispersive spectroscopy (EDS) analysis (Figure 2(c)).

2.2. Experimental Setup for SERS Analysis. The detection of $\lambda$-DNA on AgNB substrates was carried out on a confocal Raman spectrometer (Renishaw RM2000, UK). The excitation light was a focused $\mathrm{Ar}^{+}$laser (excitation wavelength $514 \mathrm{~nm}$, focal area $\sim 5 \mu \mathrm{m} \times 5 \mu \mathrm{m}$ with a $20 \times$ objective). The exposure time was $20 \mathrm{~s}$ and the detection range was $400-2000 \mathrm{~cm}^{-1}$. Figure 3 illustrates the sample preparation. As-grown AgNBs were fixed on a clean slide as the SERS substrate, and a $10 \mu \mathrm{L}$ drop of $\lambda$-DNA (Beijing Huamei Scientific Co., China) water solution was added onto the AgNBs, followed by placement of a clean $24 \mathrm{~mm} \times 24 \mathrm{~mm}$ coverslip of $0.17 \mathrm{~mm}$ thickness on the slide to seal the $\lambda$ DNA solution. Generally there would be a little spillage of the solution. The incident laser was directed vertically down and laser power on the sample was $4.7 \mathrm{~mW}$ during the analysis.

Six $\lambda$-DNA concentrations $(50,20,10,1,0.1$, and $0.01 \mathrm{ng} / \mu \mathrm{L}$ ) were examined. The average amount of $\lambda$-DNA in the detection area for each solution was estimated from the $\lambda$-DNA concentrations, the solution volume (slightly (a)
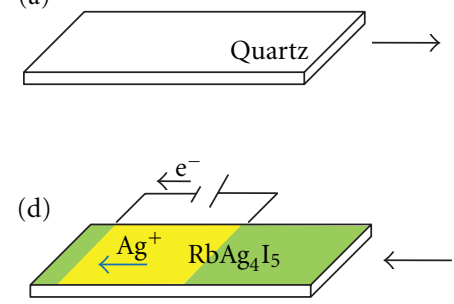

(c)

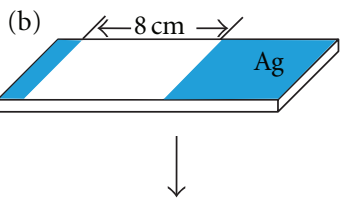

Figure 1: Fabrication of AgNB structures by superionic conductor thin film. (a) Clean quartz substrate was placed in the deposition chamber at room temperature and $10^{-4} \mathrm{~Pa}$ vacuum. (b) $1 \mu \mathrm{m}$ thick thin silver films were deposited on the quartz substrate as the electrodes. The distance between two electrodes was $8 \mathrm{~cm}$. (c) $400 \mathrm{~nm}$ thick $\mathrm{RbAg}_{4} \mathrm{I}_{5}$ superionic conductor thin film was deposited on the whole substrate as the ion-conducting medium. (d) A constant electric current was provided as the external electric field to form a directional ionic current. Silver atoms at the anode were ionized and transported to the cathode to grow into AgNB structures through the $\mathrm{RbAg}_{4} \mathrm{I}_{5}$ thin film, while electrons were transported to the cathode through external conducting wires.

smaller than $10 \mu \mathrm{L}$ due to the spillage), the surface area of the coverslip $(24 \mathrm{~mm} \times 24 \mathrm{~mm})$ and the focal area of the laser $(\sim 5 \mu \mathrm{m} \times 5 \mu \mathrm{m})$. For the $0.1 \mathrm{ng} / \mu \mathrm{L}$ solution, the average number of $\lambda$-DNA molecules in the detection area was less than 0.8 . This result indicated that the 0.1 , $0.01 \mathrm{ng} / \mu \mathrm{L}$ solutions were capable of revealing whether the AgNB substrate had single-molecule level SERS activity. The spectra of the AgNB substrate itself and a $20 \mathrm{ng} / \mu \mathrm{L} \lambda$-DNA solution on clean glass substrate without AgNB were also analyzed as controls.

\section{Results and Discussion}

SERS spectra of the six samples and two control groups are shown in Figure 4. No evident peak was seen in the red curve of $\lambda$-DNA-only control, whereas several distinct peaks were exhibited by the $20 \mathrm{ng} / \mu \mathrm{L}$ sample, for example, at 979 , 1380 , and $1594 \mathrm{~cm}^{-1}$. Such a difference provided evidence of a remarkable enhancement from the AgNB substrate. The 0.1 and $0.01 \mathrm{ng} / \mu \mathrm{L}$ solutions shared common features, including the quantity and intensities of characteristic peaks, distinguishing them from other four samples. According to the estimation result, these two groups both represented single-molecule signals, and hence the single-molecule level SERS activity of AgNB substrates has been demonstrated.

Information on secondary structures, backbone conformations and phosphate group interactions are indicated in the Raman spectra of DNA molecules. Strong and distinct characteristic peaks were all labeled in Figure 4 and their tentative assignments were presented in Table 1. Common peaks of the six solutions are approximately at 890,1045 , $1161,1380,1456,1596$, and $1694 \mathrm{~cm}^{-1}$. The 890,1161 , and $1456 \mathrm{~cm}^{-1}$ peaks reflect vibrational and deformation modes of deoxyribose. The $1045 \mathrm{~cm}^{-1}$ peak represents the phosphate group interaction, and those at 1380, 1596 and $1694 \mathrm{~cm}^{-1}$ correspond to thymine, adenine and guanine $(\mathrm{T}$, A, G) [18-23]. In addition, there are two more common 


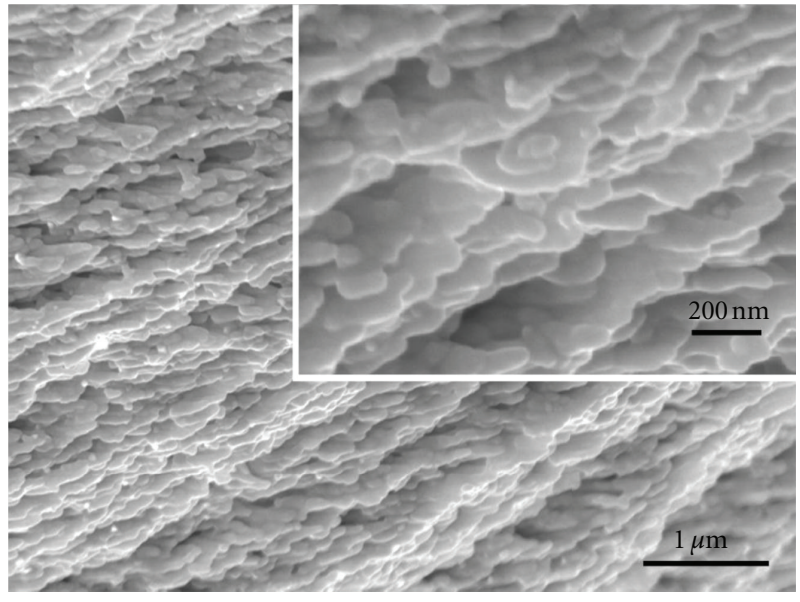

(a)

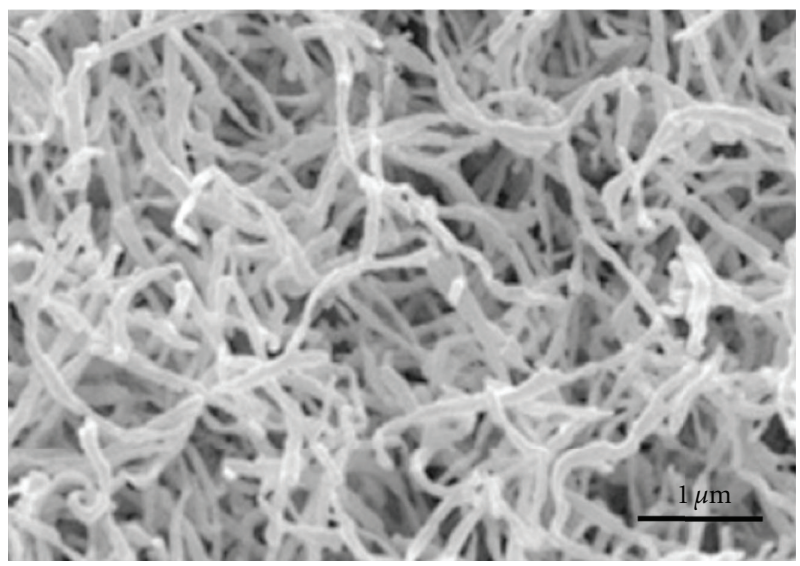

(b)

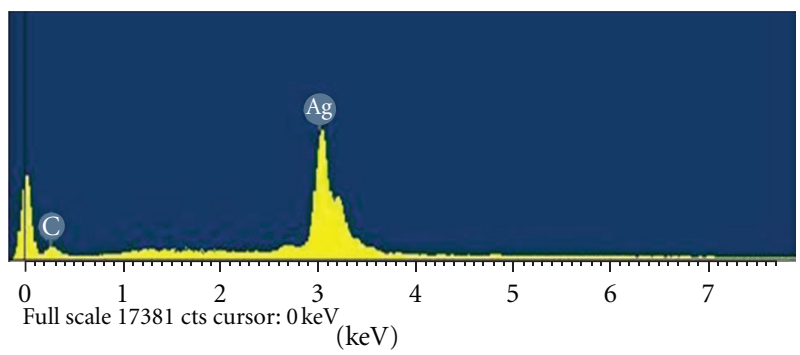

(c)

FIgure 2: SEM and EDS analyses of AgNB structures. (a) SEM image of AgNB structures (the external electric current is $12 \mu \mathrm{A}$ in the fabrication process). AgNB structures were highly rough, dense, and unidirectional, composed of tightly collocated $100 \mathrm{~nm}$ sized units like nanoscale buds growing in the same direction. (b) SEM image of AgNW structures with disordered and loose configuration obtained by decreasing the external electric current to $3 \mu \mathrm{A}$ in the fabrication process. (c) EDS analysis of AgNB structures.

peaks at 1000 and $1260 \mathrm{~cm}^{-1}$ for the $10,1,0.1$, and $0.01 \mathrm{ng} / \mu \mathrm{L}$ samples. Moreover, the single-molecule samples $(0.1$ and $0.01 \mathrm{ng} / \mu \mathrm{L})$ share another two peaks at $1285 \mathrm{~cm}^{-1}$ and $1486 \mathrm{~cm}^{-1}$. The 20 and $50 \mathrm{ng} / \mu \mathrm{L}$, samples of higher concentration have fewer common features with the four solutions of lower concentration. We conclude that the differences between these samples are mainly attributed (a)

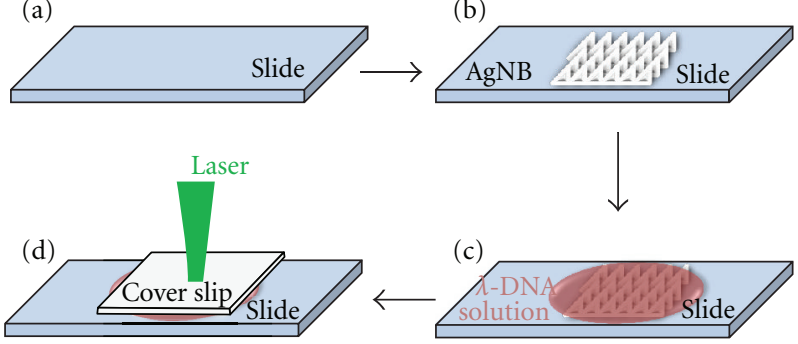

Figure 3: Preparation of $\lambda$-DNA samples on AgNB substrates. Asgrown AgNBs were fixed on a clean slide as the SERS substrate, and then a $10 \mu \mathrm{L}$ drop of $\lambda$-DNA water solution was added onto the AgNBs, thereafter a clean $24 \mathrm{~mm} \times 24 \mathrm{~mm}$ coverslip of $0.17 \mathrm{~mm}$ thickness was placed on the slide to seal the $\lambda$-DNA solution. The incident laser was directed vertically down for the analysis.

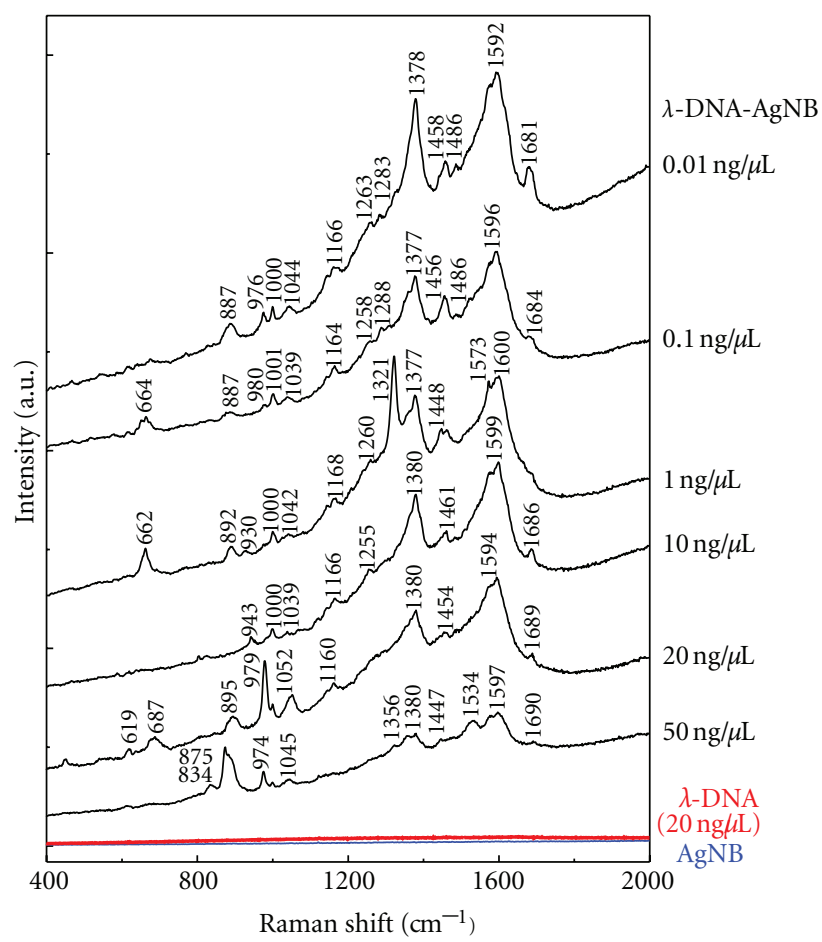

Figure 4: SERS spectra of six $\lambda$-DNA-AgNB samples (black) and two control samples (red and blue). The red spectrum was for $20 \mathrm{ng} / \mu \mathrm{L} \lambda$-DNA solution on a clean glass substrate without AgNB. The blue spectrum was for the AgNB substrate itself. The laser power on the sample was $4.7 \mathrm{~mW}$ and the exposure time was $20 \mathrm{~s}$.

to changing spatial configurations of long strand DNA molecules in water. At low concentration, the interactions between $\lambda$-DNAs are weaker, allowing $\lambda$-DNA molecules to freely extend and therefore the more similar are the Raman spectra. At higher concentration, the interactions between $\lambda$-DNAs are stronger, $\lambda$-DNA molecules are more likely to condense, markedly affecting the Raman spectra. The low concentration samples are able to display detection of a freely extended single $\lambda$-DNA molecule.

The disordered AgNW structures in Figure 2(b) are not comparable to the AgNB structures in respect of SERS 
TABLE 1: Tentative assignments for SERS spectra of $\lambda$ DNAs on AgNB substrate.

\begin{tabular}{|c|c|c|c|c|c|c|}
\hline \multicolumn{6}{|c|}{$\lambda$ DNA concentration } & \multirow{2}{*}{ Tentative assignment $\mathrm{t}^{\mathrm{a}, \mathrm{b}}$} \\
\hline $50 \mathrm{ng} / \mu \mathrm{L}$ & $20 \mathrm{ng} / \mu \mathrm{L}$ & $10 \mathrm{ng} / \mu \mathrm{L}$ & $1 \mathrm{ng} / \mu \mathrm{L}$ & $0.1 \mathrm{ng} / \mu \mathrm{L}$ & $0.01 \mathrm{ng} / \mu \mathrm{L}$ & \\
\hline & 687 & & 662 & 664 & & G \\
\hline 834 & & & & & & $\mathrm{PO}_{2}^{-}$ \\
\hline 875 & 895 & 943 & 892,930 & 887 & 887 & Deoxyribose \\
\hline 974 & 979 & 1000 & 1000 & 980,1001 & 976,1000 & Deoxyribose \\
\hline \multirow[t]{4}{*}{1045} & 1052 & 1039 & 1042 & 1039 & 1044 & $\mathrm{PO}_{2}^{-}$ \\
\hline & 1161 & 1166 & 1168 & 1164 & 1166 & Deoxyribose \\
\hline & & 1255 & 1260 & 1258 & 1263 & T, C, A \\
\hline & & & & 1288 & 1283 & $\mathrm{C}, \mathrm{A}$ \\
\hline 1356 & & & 1321 & & & $\mathrm{~A}, \mathrm{G}$ \\
\hline 1380 & 1380 & 1380 & 1377 & 1377 & 1378 & $\mathrm{~T}, \mathrm{~A}, \mathrm{G}$ \\
\hline 1447 & 1454 & 1461 & 1448 & 1456,1486 & 1458,1486 & Deoxyribose, $\mathrm{CH}_{2}$ \\
\hline 1534 & & & & & & $\mathrm{~A}$ \\
\hline 1597 & 1594 & 1599 & 1573,1600 & 1596 & 1592 & $A, G$ \\
\hline 1690 & 1689 & 1686 & & 1684 & 1681 & $\mathrm{~T}$ \\
\hline
\end{tabular}

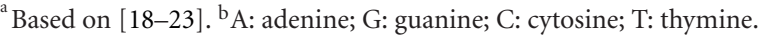

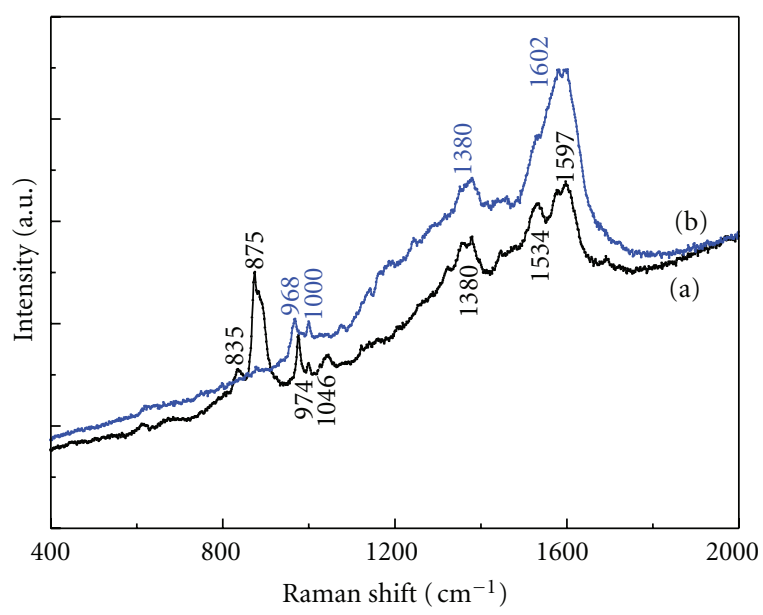

(a) AgNB substrate

(b) Disordered AgNW substrate

Figure 5: SERS spectra of $\lambda$-DNA samples $(50 \mathrm{ng} / \mu \mathrm{L})$ on the AgNB substrate (a) and on the disordered AgNW substrate (b). Characteristic peaks of $\lambda$-DNA on the AgNB substrate were more distinguishable than those on the disordered AgNW substrate, with relatively high intensity and low fluorescence background.

activity. Spectra of $\lambda$-DNA samples $(50 \mathrm{ng} / \mu \mathrm{L})$ on the AgNB substrate and on the disordered AgNW substrate are displayed in Figure 5. The characteristic peaks of $\lambda$-DNA on the AgNB substrate were more distinguishable than those on the disordered AgNW substrate, with relatively high intensity and low fluorescence background. In addition, the spectrum obtained on the AgNB substrate had seven distinct peaks, while that found with the disordered AgNW substrate only had four.

SERS activity is strongly dependent on the surface topography of the substrate. It is demonstrated in hotspot theories that the localized field enhancement is more intense with more hot-spots on the surface of noble metal materials, and so the amount of hot-spots is related to SERS activity [24-27]. The AgNB substrate has nanoscale roughness and a unidirectional dense structure, making it a perfect environment for hot-spots and the generation of significant localized field enhancements. The disordered AgNW substrate only has microscale roughness and a loose structure, precluding the generation of sufficient hot-spots to bring about comparable SERS activity. The results demonstrate that most of the excitation energy was transferred into Raman scattering of detected molecules on the AgNB substrate through SPR effects, and the signal-to-noise ratio was heightened.

\section{Conclusions}

In this letter, we have reported a highly rough and unidirectional AgNB substrate with excellent SERS activity. We have shown that AgNB substrates are capable of detecting $\lambda$-DNA at the single-molecule level, and discussed the SERS spectra of $\lambda$-DNA molecules. We have also demonstrated that such dense and unidirectional AgNB structures are better SERS substrates than similarly made loose and disordered AgNW structures. This novel AgNB substrate is associated with a much lower detection limit than former SERS substrates, providing an efficient and reliable approach for biomedical analysis.

\section{Acknowledgments}

This work was supported by the National Nature Science Foundation of China (Grants no. 90919012, 10874099, and 11174172), the Doctoral Program Research Fund of Chinese Ministry of Education (Grant no. 20090002110065) and Tsinghua University Initiative Scientific Research Program (2010THZ01). 


\section{References}

[1] Y. W. C. Cao, R. Jin, and C. A. Mirkin, "Nanoparticles with Raman spectroscopic fingerprints for DNA and RNA detection," Science, vol. 297, no. 5586, pp. 1536-1540, 2002.

[2] R. Tuma, "Raman spectroscopy of proteins: from peptides to large assemblies," Journal of Raman Spectroscopy, vol. 36, no. 4, pp. 307-319, 2005.

[3] G. Haran, "Single-molecule raman spectroscopy: a probe of surface dynamics and plasmonic fields," Accounts of Chemical Research, vol. 43, no. 8, pp. 1135-1143, 2010.

[4] J. H. Kim, M. Kataoka, D. Shimamoto et al., "Raman and fluorescence spectroscopic studies of a DNA-dispersed double-walled carbon nanotube solution," ACS Nano, vol. 4, no. 2, pp. 1060-1066, 2010.

[5] C. Mallidis, J. Wistuba, B. Bleisteiner et al., "In situ visualization of damaged DNA in human sperm by Raman microspectroscopy," Human Reproduction, vol. 26, no. 7, pp. 1641-1649, 2011.

[6] J. Gersten and A. Nitzan, "Electromagnetic theory of enhanced Raman scattering by molecules adsorbed on rough surfaces," The Journal of Chemical Physics, vol. 73, no. 7, pp. 3023-3027, 1980.

[7] K. Kneipp, Y. Wang, H. Kneipp et al., "Single molecule detection using surface-enhanced Raman scattering (SERS)," Physical Review Letters, vol. 78, no. 9, pp. 1667-1670, 1997.

[8] S. Nie and S. R. Emory, "Probing single molecules and single nanoparticles by surface-enhanced Raman scattering," Science, vol. 275, no. 5303, pp. 1102-1106, 1997.

[9] H. Xu, E. J. Bjerneld, M. Käll, and L. Börjesson, "Spectroscopy of single hemoglobin molecules by surface enhanced Raman scattering," Physical Review Letters, vol. 83, no. 21, pp. 43574360, 1999.

[10] M. Kahl and E. Voges, "Analysis of plasmon resonance and surface-enhanced Raman scattering on periodic silver structures," Physical Review B, vol. 61, no. 20, pp. 14078 14088, 2000.

[11] K. Kneipp, H. Kneipp, and J. Kneipp, "Surface-enhanced raman scattering in local optical fields of silver and gold nanoaggregates-from single-molecule raman spectroscopy to ultrasensitive probing in live cells," Accounts of Chemical Research, vol. 39, no. 7, pp. 443-450, 2006.

[12] E. J. Blackie, E. C. Le Ru, and P. G. Etchegoin, "Singlemolecule surface-enhanced raman spectroscopy of nonresonant molecules," Journal of the American Chemical Society, vol. 131, no. 40, pp. 14466-14472, 2009.

[13] J. A. Dieringer, K. L. Wustholz, D. J. Masiello et al., "Surface-enhanced Raman excitation spectroscopy of a single rhodamine 6G molecule," Journal of the American Chemical Society, vol. 131, no. 2, pp. 849-854, 2009.

[14] S. Rao, S. Raj, S. Balint et al., "Single DNA molecule detection in an optical trap using surface-enhanced Raman scattering," Applied Physics Letters, vol. 96, no. 21, Article ID 213701, 2010.

[15] Y. Cao, H. S. Sun, J. L. Sun, G. Y. Tian, Z. Xing, and J. H. Guo, "Preparation and structural characterization of superionic conductor RbAg4I5 crystalline grain film," Chinese Physics Letters, vol. 20, no. 5, pp. 756-758, 2003.

[16] S. Shi, J. Sun, G. Zhang, J. Guo, and Z. Wang, "The growth of thin silver nanowires bundle using RbAg4I 5 crystal grain thin film and the ionic conductivity of the thin film," Physica B, vol. 362, no. 1-4, pp. 266-270, 2005.

[17] J. L. Sun, J. H. Zhang, W. Liu et al., "Shape-controlled synthesis of silver nanostructures," Nanotechnology, vol. 16, no. 10, pp. 2412-2414, 2005.
[18] W. L. Peticolas, "Raman spectroscopy of DNA and proteins," Methods in Enzymology, vol. 246, pp. 389-416, 1995.

[19] H. Deng, V. A. Bloomfield, J. M. Benevides, and G. Thomas, "Dependence of the Raman signature of genomic B-DNA on nucleotide base sequence," Biopolymers, vol. 50, no. 6, pp. 656666, 1999.

[20] H. Wei and H. Xu, "Surface-enhanced Raman scattering of $\lambda$ -DNA," Applied Physics A, vol. 89, no. 2, pp. 273-275, 2007.

[21] J. De Gelder, K. De Gussem, P. Vandenabeele, and L. Moens, "Reference database of Raman spectra of biological molecules," Journal of Raman Spectroscopy, vol. 38, no. 9, pp. 1133-1147, 2007.

[22] L. L. Sun, Y. J. Sun, F. G. Xu et al., "Atomic force microscopy and surface-enhanced raman scattering detection of DNA based on DNA-nanoparticle complexes," Nanotechnology, vol. 20, no. 12, Article ID 125502, 2009.

[23] C. M. Muntean, N. Leopold, A. Halmagyi, and S. Valimareanu, "Surface-enhanced Raman spectroscopy of DNA from leaves of in vitro grown apple plants," Journal of Raman Spectroscopy, vol. 42, no. 4, pp. 844-850, 2011.

[24] A. M. Michaels, J. Jiang, and L. Brus, "Ag Nanocrystal Junctions as the Site for Surface-Enhanced Raman Scattering of Single Rhodamine 6G Molecules," Journal of Physical Chemistry B, vol. 104, no. 50, pp. 11965-11971, 2000.

[25] J. Margueritat, H. Gehan, J. Grand et al., "Influence of the number of nanoparticles on the enhancement properties of surface-enhanced raman scattering active area: sensitivity versus repeatability," ACS Nano, vol. 5, no. 3, pp. 1630-1638, 2011.

[26] A. Lee, G. F. S. Andrade, A. Ahmed et al., "Probing dynamic generation of hot-spots in self-assembled chains of gold nanorods by surface-enhanced raman scattering," Journal of the American Chemical Society, vol. 133, no. 19, pp. 7563-7570, 2011.

[27] N. J. Borys and J. M. Lupton, "Surface-enhanced light emission from single hot spots in tollens reaction silver nanoparticle films: linear versus nonlinear optical excitation," Journal of Physical Chemistry C, vol. 115, no. 28, pp. 1364513659, 2011. 

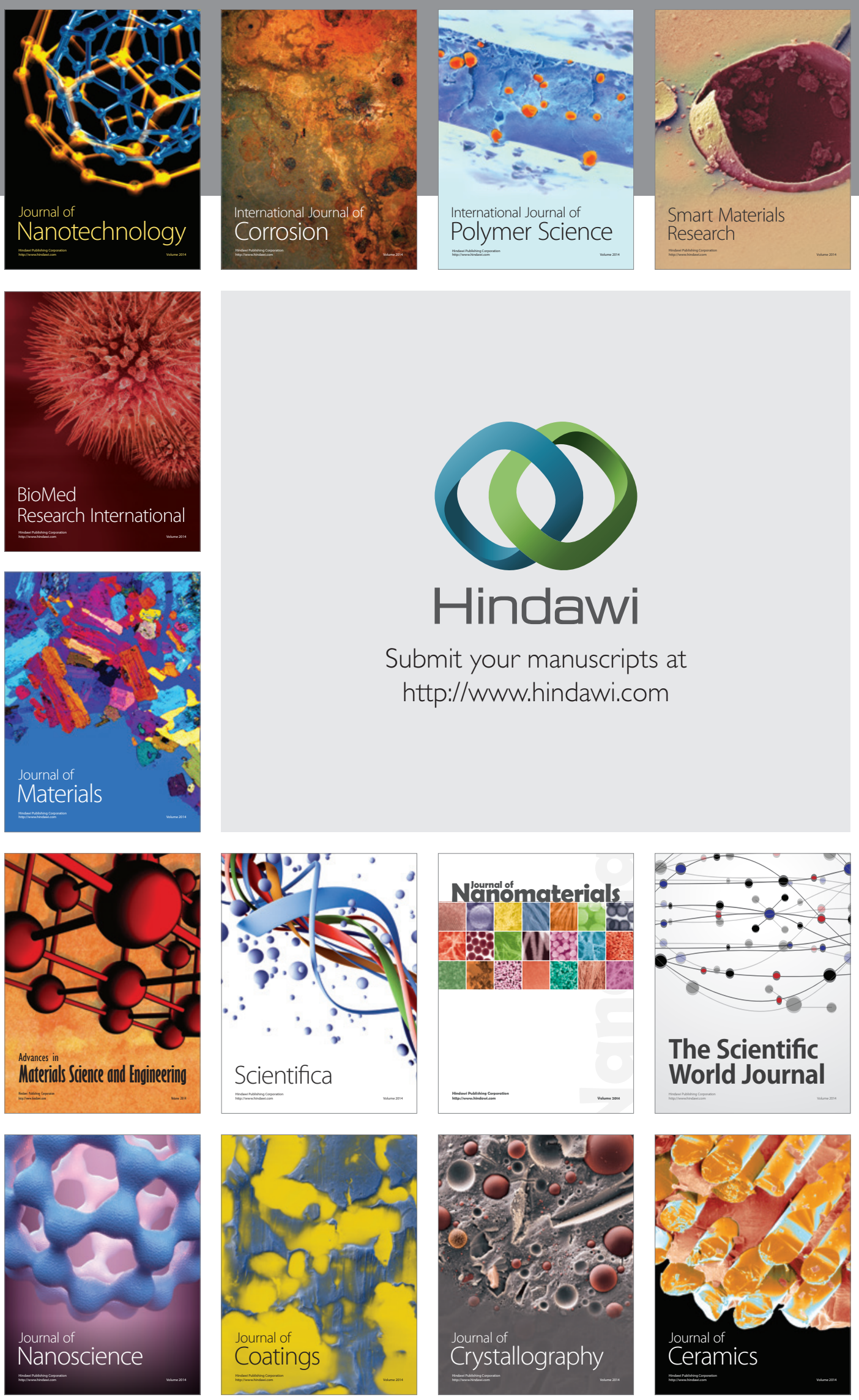

The Scientific World Journal

Submit your manuscripts at

http://www.hindawi.com

\section{World Journal}

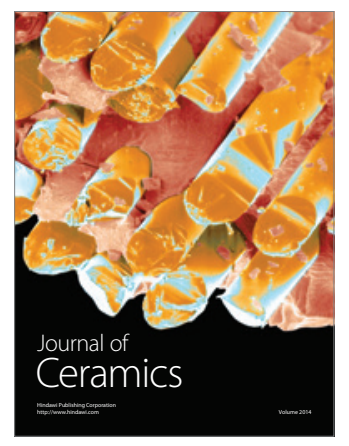

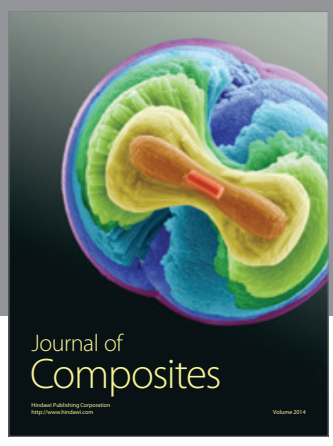
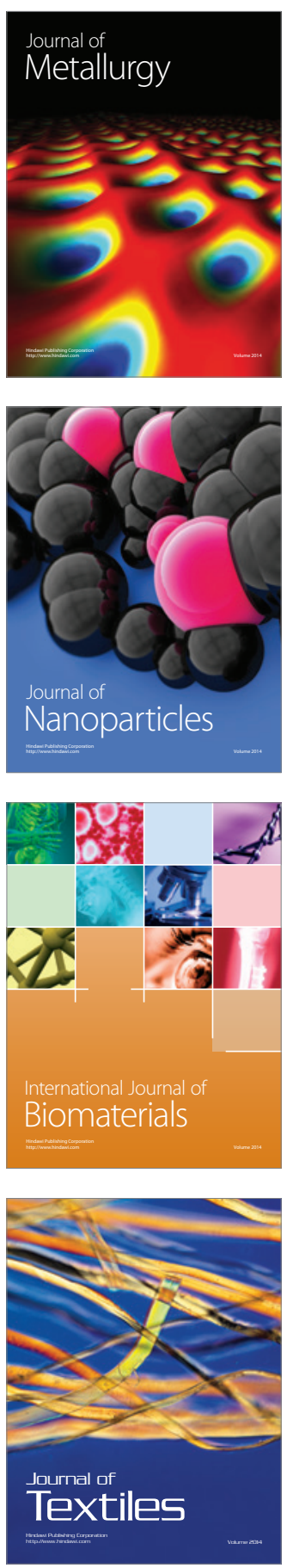